\title{
Dynamique des populations de tiques parasites des bovins de la région du Gharb au Maroc
}

\author{
M. Laamri ${ }^{1 *}$ K. El Kharrim ${ }^{1}$ R. Mrifag ${ }^{1}$ \\ M. Boukbal ${ }^{1}$ D. Belghyti ${ }^{1}$
}

\section{Mots-clés}

Bovin - Metastigmata -

Développement saisonnier - Lutte antiacarien - Gharb - Maroc.

\begin{abstract}
Résumé
Cette étude a été conduite dans la commune rurale de Sidi Boubker El Haj, région du Gharb (Maroc), de novembre 2009 à octobre 2010. L'objectif a été d'identifier les différentes espèces de tiques rencontrées chez les bovins, de suivre leur dynamique saisonnière, et de déterminer leur abondance et la variabilité du niveau d'infestation selon l'âge et le sexe des hôtes. Trente bovins ont fait l'objet de suivi et de prélèvements mensuels pendant une année. Au total, 6899 tiques ont été récoltées. L'identification a révélé la présence de neuf espèces appartenant à cinq genres, avec les prévalences suivantes : Rhipicephalus bursa (28,61 p. 100), Ixodes ricinus (26,39 p. 100), Hyalomma lusitanicum (23,33 p. 100), Rhipicephalus turanicus (14,44 p. 100), Hyalomma detritum detritum (13,06 p. 100), Rhipicephalus sanguineus (1,39 p. 100), Hyalomma marginatum marginatum (1,11 p. 100), Dermacentor marginatus (1,11 p. 100) et Haemaphysalis sulcata $(0,83$ p. 100). I. ricinus a présenté un mode d'activité unimodal hivernal, alors que $R$. bursa, Hyalomma lusitanicum et Rhipicephalus turanicus ont été actives au printemps, et Hyalomma $d$. detritum en été. $R$. sanguineus, Hyalomma m. marginatum, D. marginatus et Haemaphysalis sulcata ont été moins prévalentes, leur présence sur le bétail pouvant être qualifiée de parasitisme occasionnel. Cette étude sur la dynamique des populations de tiques peut aider à la mise en place d'une stratégie adaptée de lutte contre les tiques et les maladies qu'elles transmettent, en tenant compte de l'activité saisonnière des tiques, de la charge parasitaire, et du taux d'infestation.
\end{abstract}

\section{INTRODUCTION}

Les tiques sont des ectoparasites obligatoires qui se nourrissent du sang des vertébrés, particulièrement celui des mammifères et des oiseaux. Les morsures de tiques peuvent être directement débilitantes pour les animaux domestiques provoquant l'hypersensibilité, l'irritation, l'inflammation et des dommages physiques. Lorsqu'elles sont présentes en grand nombre, les tiques peuvent provoquer une anémie et une réduction de la productivité. Les sécrétions salivaires de certaines espèces peuvent causer la toxicose et la paralysie. Les tiques peuvent également transmettre au bétail un grand nombre de maladies virales, rickettsiales et

1. Laboratoire d'environnement et énergies renouvelables, UFR Parasitologie comparée, faculté des Sciences, Université Ibn Tofail, BP 133, CP 14000, Kenitra, Maroc.

*Auteur pour la correspondance

Tél. : +212668995787; fax : + 212537329433

E-mail : laamri24@hotmail.com bactériennes (20). Les maladies associées aux tiques ont un impact important sur la productivité des animaux, associé à des pertes économiques pour leurs propriétaires. Elles continuent d'être un obstacle majeur à l'amélioration de l'élevage en Afrique, car ce continent est affecté par un grand nombre d'espèces de tiques et par les diverses maladies associées ou transmises (19).

La dynamique saisonnière des tiques conditionne la transmission des agents pathogènes (18). Les préférences écologiques des tiques sont variables, chaque espèce ayant besoin de conditions environnementales particulières lui permettant de vivre dans un biotope donné, ce qui influence sa distribution géographique. Plusieurs facteurs écologiques influencent la survie et le développement des tiques, en particulier la température, l'humidité relative et le couvert végétal (18).

Au Maroc, la région du Gharb, à vocation agricole et d'élevage, n'est pas épargnée. Le cheptel local subit de lourdes pertes en raison des infections transmises par les tiques et les plus grands 
dommages sont causés par Anaplasma, Babesia et Theileria. La prévalence de babésioses à $B$. bovis et à $B$. bigemina, et de la theilériose à $T$. annulata, dans la région du Gharb, sont respectivement de 25,23 et 19 p. 100 (18).

Les études bioécologiques des tiques sont nécessaires pour établir des programmes de lutte contre ces parasites. Ces études passent par une bonne identification des tiques pour permettre l'établissement de la liste de la faune ixodidienne parasitant les troupeaux d'une région, la description de leur écologie et de leur distribution (2). Cela permet aussi de préciser les espèces qui jouent un rôle vecteur et par conséquent de mieux cibler la lutte (2).

La présente étude a eu pour objectif de suivre, au cours d'une année, l'évolution de l'infestation des bovins par les différentes espèces de tiques rencontrées dans la commune de Sidi Boubker El Haj, dans la région du Gharb, de déterminer l'abondance de ces espèces et la variabilité du niveau d'infestation selon l'âge et le sexe des hôtes.

\section{MATERIEL ET METHODES}

\section{Région d'étude}

La commune de Sidi Boubker El Haj fait partie des communes rurales de la région du Gharb (figure 1). Elle est délimitée au nord par Larache, à l'ouest par l'océan Atlantique et à l'est par la ville d'Ouezzane. Elle est située à 102 mètres en moyenne au dessus du niveau de la mer. Sa superficie totale est de 15500 ha. Cette commune rurale bénéficie d'un climat méditerranéen doux et subhumide, caractérisé par une pluviométrie moyenne annuelle comprise entre $600 \mathrm{~mm}$ et $900 \mathrm{~mm}$, et une température moyenne de $16,7^{\circ} \mathrm{C}$ (minimum hivernal de $5,5^{\circ} \mathrm{C}$; maximum estival de $27,9^{\circ} \mathrm{C}$ ) (12). La saison hivernale s'étend d'octobre à avril, et la saison estivale de juin à septembre.

La végétation est caractérisée par une couverture forestière abondante constituée en majeure partie de chênes-lièges, d'eucalyptus, de cistes et de fougères. Sur le plan économique, les ressources de la commune proviennent essentiellement de l'élevage et de l'agriculture. En matière d'élevage, le cheptel bovin est assez développé dans la région et représente 8,9 p. 100 de la production animale nationale. L'élevage est généralement de type semi-intensif et extensif. Les animaux sont nourris au foin, à la luzerne et à l'herbe pendant la belle saison $(10,12)$.

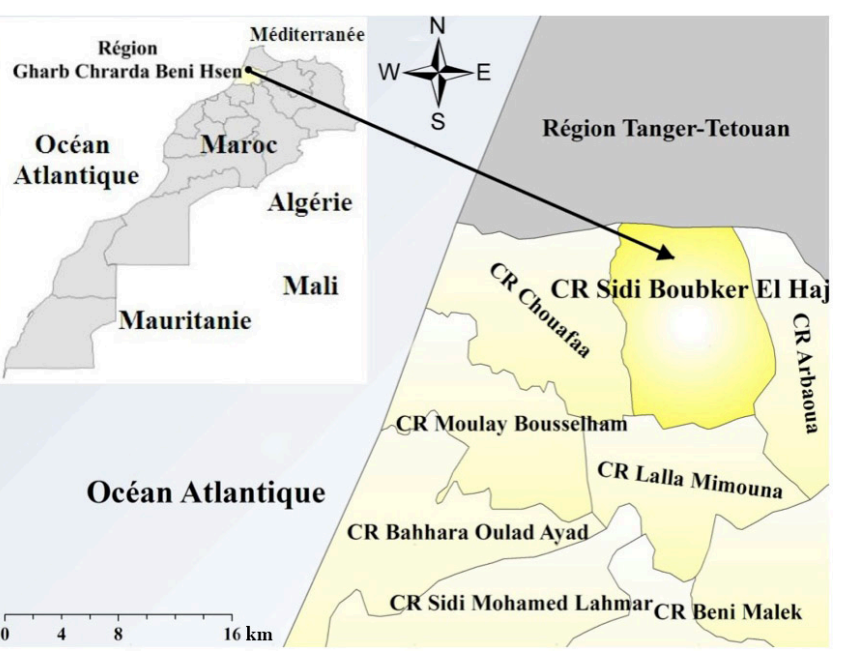

Figure 1 : situation géographique de la zone d'étude. $C R$ : commune rurale.

\section{Animaux et rythmes de prélèvement}

Trente bovins des deux sexes ont été suivis pendant une année, de novembre 2009 à octobre 2010. Tous les animaux appartenaient à un élevage extensif. Pendant toute la durée de l'étude, l'échantillon a été le même, sans remplacement ou perte. Les pâturages utilisés ont été fréquentés par d'autres troupeaux, tels que les ovins et les caprins. Aucun traitement acaricide n'a été administré aux animaux. Les tiques ont été récoltées une fois par mois pendant 12 mois.

\section{Collecte des tiques}

Les tiques ont été collectées par régions anatomiques et conservées dans des flacons à fermeture hermétique contenant du formol à 10 p. 100. Les flacons ont été identifiés par les mentions suivantes : numéro, sexe et âge du bovin, numéro d'ordre de l'échantillon, date de récolte, zone anatomique.

\section{Identification des tiques}

L'identification des tiques a reposé sur des caractéristiques morpho-anatomiques en se basant sur les clés d'indentification des tiques $(2,17,19)$. Cette identification a été réalisée sous une loupe binoculaire (DM 143 Motic Digital Microscope, UE) au Laboratoire d'environnement et des énergies renouvelables de la faculté des Sciences à Kénitra. L'observation des caractéristiques morphologiques externes a été faite en montant les différents segments des tiques entre lame et lamelle dans l'acide lactique, observés à l'aide d'un microscope optique (Optika, B-350). La technique du découpage du gonopore a été utilisée pour identifier les femelles des genres Rhipicephalus et Hyalomma (2). Le microscope électronique à balayage (Quanta 200) a été utilisé pour confirmer l'identification au Centre national de recherches scientifiques et techniques de Rabat (CNRST) (5).

\section{Analyses statistiques}

Les données collectées pendant la période d'étude ont été enregistrées selon le sexe et le stade de développement des tiques. Les indices parasitaires ont été calculés selon Margolis et coll. (11). Pour chaque espèce : la prévalence, l'abondance et l'intensité parasitaire moyenne ont été déterminées, ainsi que les moyennes mensuelles accompagnées des écarts-types.

\section{Prévalence}

La prévalence $(\mathrm{P})$ est le rapport en pourcentage du nombre d'hôtes infestés $(\mathrm{N})$ par une espèce de parasite donnée sur le nombre d'hôtes examinés $(\mathrm{H})$.

$\mathrm{P}(\%)=\mathrm{N} / \mathrm{H} * 100$

\section{Abondance}

L'abondance (A) correspond au rapport du nombre total d'individus d'une espèce parasite (n) sur le nombre total des individus examinés $(\mathrm{H})$.

$\mathrm{A}=\mathrm{n} / \mathrm{H}$

\section{Intensité parasitaire moyenne}

L'intensité parasitaire moyenne (I) correspond au rapport du nombre total d'individus d'une espèce parasite (n) dans un échantillon d'hôtes sur le nombre d'hôtes infestés $(\mathrm{N})$ dans l'échantillon.

$\mathrm{I}=\mathrm{n} / \mathrm{N}$ 


\section{RESULTATS ET DISCUSSION}

Au total 6899 tiques ont été récoltées au cours de 12 collectes. Le tableau I montre les espèces identifiées en fonction du sexe et du stade de développement. Le tableau II montre que $R$. bursa a présenté la plus forte prévalence, et $H$. $d$. detritum la plus forte abondance et intensité parasitaire. Les tiques ont présenté une activité saisonnière avec une période d'infestation plus ou moins limitée selon les espèces.

Tous les stades de $R$. bursa ont parasité les bovins, cette espèce ayant un cycle monotrope. Les nymphes ont été récoltées de novembre 2009 à mai 2010. Le pic d'infestation a été observé en janvier (8,7 tiques/bovin). Les larves ont été observées de décembre 2009 à mars 2010 avec un pic d'infestation en janvier (12,7 tiques/ bovin). Les adultes sont apparus en avril et ont disparu en août avec un pic d'infestation en mai (4,37 tiques/bovin) (figure 2). $R$. bursa a présenté une prévalence élevée et une intensité parasitaire moyenne faible, indiquant que cette espèce était distribuée de manière homogène. En 2007, Sahibi et Rhalem ont noté que la prévalence de cette espèce était de 13 p. 100 dans la région de Gharb (18). Les travaux menés par Morel ont révélé que la dynamique saisonnière de $R$. bursa est unimodale, avec un seul pic d'activité pendant la saison chaude (de mars à septembre avec un maximum en juin) et celle des immatures s'étendait d'octobre à mars (14). R. bursa a développé un cycle particulier à deux hôtes diphasique monotrope et exophile (3), avec suppression de la phase libre entre la larve et la nymphe, et métamorphose sur l'organisme hôte (6). $R$. bursa a été récoltée au Maroc, en Algérie, en Tunisie et en Libye, dans les zones bioclimatiques humides, subhumides et semi-arides (2).

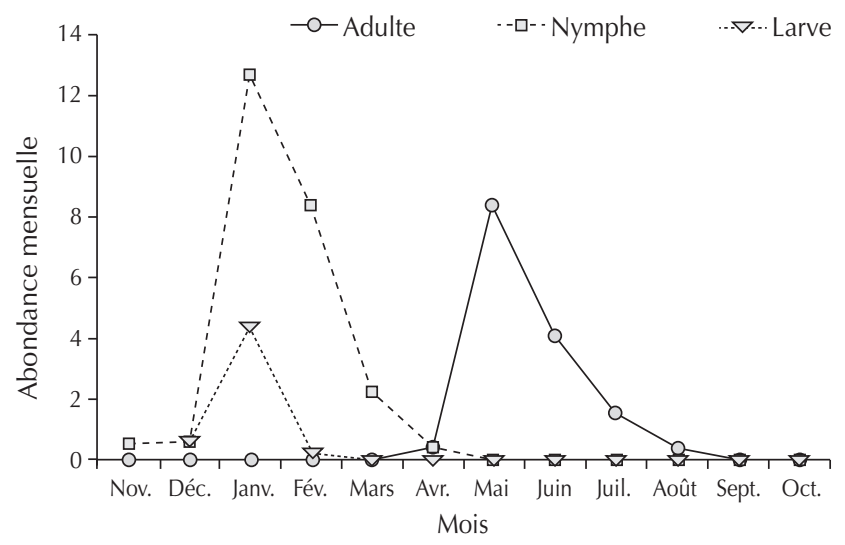

Figure 2 : fluctuations de l'abondance mensuelle de Rhipicephalus bursa adulte, nymphe et larve dans la commune rurale de Sidi Bouker Elhaj dans la région du Charb au Maroc.

\section{Tableau I}

Espèces de tiques récoltées en fonction du sexe et du stade de développement

$\begin{array}{lrrrrr}\text { Espèce de parasite } & \text { Mâle } & \text { Femelle } & \text { Nymphe } & \text { Larve } & \text { Nb. total d'individus } \\ \text { Ixodes ricinus (Linnaeus, 1758) } & 254 & 328 & - & - & 582 \\ \text { Rhipicephalus bursa (Canestrini et Fanzago, 1878) } & 233 & 203 & 748 & 142 & 1326 \\ \text { Hyalomma detritum detritum (Schulze, 1919) } & 1588 & 593 & 1245 & 436 & 3862 \\ \text { Hyalomma lusitanicum (Koch, 1844) } & 619 & 280 & - & - & 899 \\ \text { Rhipicephalus turanicus (Pomerantsev, 1936) } & 93 & 95 & 5 & - & 193 \\ \text { Rhipicephalus sanguineus (Latreille, 1806) } & 10 & 11 & - & - & -21 \\ \text { Hyalomma marginatum marginatum (Koch, 1844) } & 7 & 2 & - & - & 9 \\ \text { Haemaphysalis sulcata (Canestrini et Fanzago, 1878) } & 2 & 1 & - & - & 4 \\ \text { Dermacentor marginatus (Sulzer, 1776) } & 2 & 2 & - & 578 & 689\end{array}$

\section{Tableau II}

Prévalence, abondance et intensité parasitaire moyennes annuelles des espèces de tiques parasites des bovins

\begin{tabular}{|c|c|c|c|c|c|}
\hline Parasite & $\begin{array}{l}\text { Hôte infesté } \\
\text { annuellement } \\
\text { par espèce }\end{array}$ & $\begin{array}{l}\text { Nb. d'hôtes infestés } \\
\text { au moins une fois } \\
\text { au cours du suivi }\end{array}$ & Prévalence (\%) & Abondance & $\begin{array}{c}\text { Intensité parasitaire } \\
\text { moyenne }\end{array}$ \\
\hline Rhipicephalus bursa & 103 & 30 & 28,61 & 3,69 & 12,89 \\
\hline Ixodes ricinus & 95 & 30 & 26,39 & 1,62 & 6,13 \\
\hline Hyalomma lusitanicum & 84 & 30 & 23,33 & 2,50 & 10,70 \\
\hline Rhipicephalus turanicus & 52 & 27 & 14,44 & 0,54 & 3,71 \\
\hline Hyalomma d. detritum & 47 & 24 & 13,06 & 10,73 & 82,17 \\
\hline Rhipicephalus sanguineus & 5 & 5 & 1,39 & 0,06 & 4,20 \\
\hline Hyalomma m. marginatum & 4 & 3 & 1,11 & 0,03 & 2,25 \\
\hline Dermacentor marginatus & 4 & 3 & 1,11 & 0,01 & 1,00 \\
\hline Haemaphysalis sulcata & 3 & 3 & 0,83 & 0,01 & 1,00 \\
\hline
\end{tabular}


Cette espèce est le vecteur de Babesia bovis, B. bigemina et Anaplasma marginale chez les bovins. C'est une espèce commune chez le bétail dans la région méditerranéenne (18).

I. ricinus a été récoltée de novembre 2009 à mai 2010, avec un pic en janvier (7,5 tiques/bovin) (figure 3). Elle a présenté une prévalence forte, une intensité parasitaire moyenne faible, révélant une homogénéité de sa distribution. Seuls les adultes ont parasité les bovins. Dans cette étude, elle a présenté un mode d'activité unimodal hivernal qui se rencontre surtout dans des conditions climatiques défavorables (1). Dans les pays tempérés d'Europe, la population active d'I. ricinus évolue de manière bimodale au cours de l'année avec un pic important au printemps et un second pic moins important en automne (7). I. ricinus parasite fréquemment les bovins et rarement les ovins. Elle a été récoltée au Maroc, en Algérie et en Tunisie, dans les zones bioclimatiques humides et subhumides (2). C'est une espèce mésophile qui, pour s'adapter au climat méditerranéen chaud de l'Afrique du Nord, a inversé son rythme d'activité saisonnière par rapport à l'Europe et s'est localisée aux stations les plus humides (15).

Les adultes d'Hyalomma lusitanicum ont parasité les bovins d'avril à octobre 2010, avec une forte charge parasitaire en avril (15 tiques/ bovin) (figure 3 ). Elle a présenté une prévalence élevée et une faible intensité parasitaire moyenne, révélant une distribution homogène. C'est une espèce à cycle triphasique, ditrope, dont la distribution est liée à la présence des rongeurs sur lesquels se gorgent les stades immatures $(12,15)$. Elle peut être abondante dans les zones où les bovins et les lapins sont les seuls hôtes disponibles (8). Les stades immatures se retrouvent sur de petits animaux, y compris les lapins (18). Au Maroc, cette espèce se rencontre dans les régions bioclimatiques subhumides à arides à hiver tempéré (16). Elle a été rapportée comme le vecteur naturel de Theileria annulata en Espagne (8).

$R$. turanicus a été récoltée entre mars et août 2010 avec une forte charge parasitaire en avril (3,2 tiques/bovin) (figure 3). Les indices parasitaires de $R$. turanicus ont été faibles, indiquant que le parasitisme par cette espèce a été de faible incidence. $R$. turanicus est apparue sur les bovins en mars et a disparu en août avec une forte charge parasitaire observée en avril. Elle a été récoltée au Maroc, en Algérie, en Tunisie et en Libye, dans les zones bioclimatiques humides à arides (2). C'est une espèce printanière qui apparaît en mars et disparaît en juillet (15).

Hyalomma detritum detritum a été observée sous ses trois stades évolutifs. Les larves ont été récoltées en septembre et octobre, et les nymphes de septembre à novembre. Les adultes de cette espèce étaient présents de mai à août 2010, avec un pic en juin

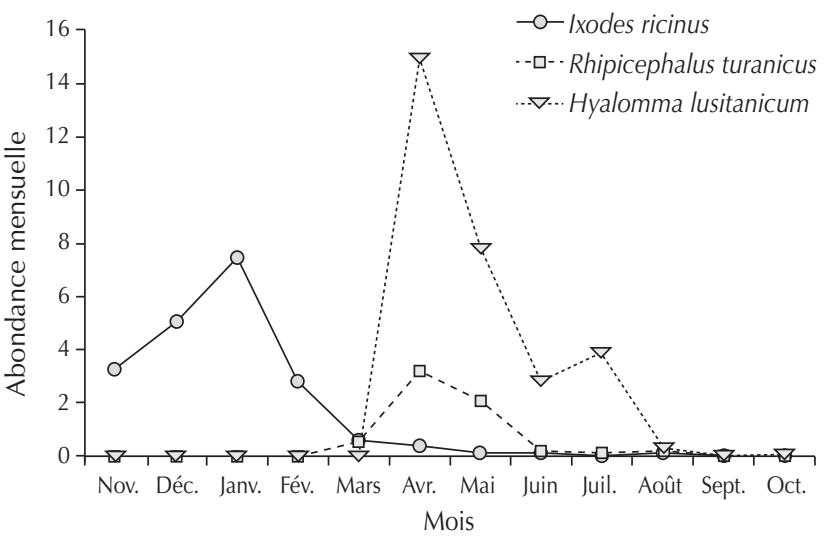

Figure 3 : fluctuations de l'abondance mensuelle d'Ixodes ricinus, de Rhipicephalus turanicus et de Hyalomma lusitanicum adulte dans la commune rurale de Sidi Boubker Elhaj dans la région du Gharb au Maroc.
(64,16 tiques/bovin) (figure 4). Elle a présenté une intensité parasitaire moyenne élevée et une prévalence faible. Un effectif de tiques très important a été récolté sur un nombre réduit d'hôtes, ce qui est appelé « phénomène d'agrégation parasitaire » et peut être expliqué par les infestations survenues dans les étables. Hyalomma d. detritum se rencontre presque exclusivement sur les bêtes qui dorment à l'étable toute l'année (21). Des travaux menés par les chercheurs de l'Institut agronomique et vétérinaire Hassan II ont indiqué que la prévalence de cette espèce est de 18 p. 100 dans la région de Gharb (18). Elle se rencontre dans les étables, les fentes, les crevasses des murs, les rochers et les pierrailles (14). Les résultats de la présente étude ont montré que les trois stades de Hyalomma d. detritum ont parasité les bovins (tique monotrope). Au cours du cycle annuel, les adultes ont eu une activité estivale, de mai à août, avec une forte charge parasitaire en juin. Ce résultat confirme le cycle diphasique monotrope observé par Sahibi et Rhalem chez ces tiques au Maroc. Les bovins domestiques sont les hôtes habituels de cette espèce (18). Les adultes de Hyalomma d. detritum apparaissent à la fin du printemps avec un pic en juillet puis disparaissent début septembre. Les larves et les nymphes se gorgent sur les bovins en automne de septembre à novembre (18). Hyalomma d. detritum est le principal vecteur de la theilériose à Theileria annulata dans certaines zones d'Afrique du Nord (18). Bouattour a indiqué que Hyalomma d. detritum est largement distribuée dans les étages bioclimatiques humides, subhumides et semi-arides (2).

Les prévalences des espèces $R$. sanguineus, Hyalomma marginatum marginatum, $D$. marginatus et $H$. sulcata ont été très faibles sur le bétail, indiquant un parasitisme occasionnel pour ces espèces. L'infestation par $R$. sanguineus dans cette zone d'étude a été très réduite. D'après Yousfi-Monod et Aeschlimann (21), le bétail parasité est en général celui qui cohabite avec les chiens domestiques. C'est une espèce nettement thermophile.

Quelques spécimens seulement de Hyalomma m. marginatum ont été récoltés dans cette région, et ce, en raison des conditions climatiques. Les fortes précipitations des deux dernières années ont rendu le climat local subhumide (10). Ouhelli signale que Hyalomma m. marginatum est largement distribuée dans les zones bioclimatiques humides à arides (16). Elle est incriminée dans la transmission de la theilériose bovine à $T$. annulata, des rickettsioses et des babésioses (16).

Dans la présente étude, l'infestation par Dermacentor marginatus a été faible. C'est une espèce à cycle triphasique ditrope, avec une endophilie des stades immatures qui parasitent les micromammifères, et une exophilie du stade adulte qui parasite essentiellement les

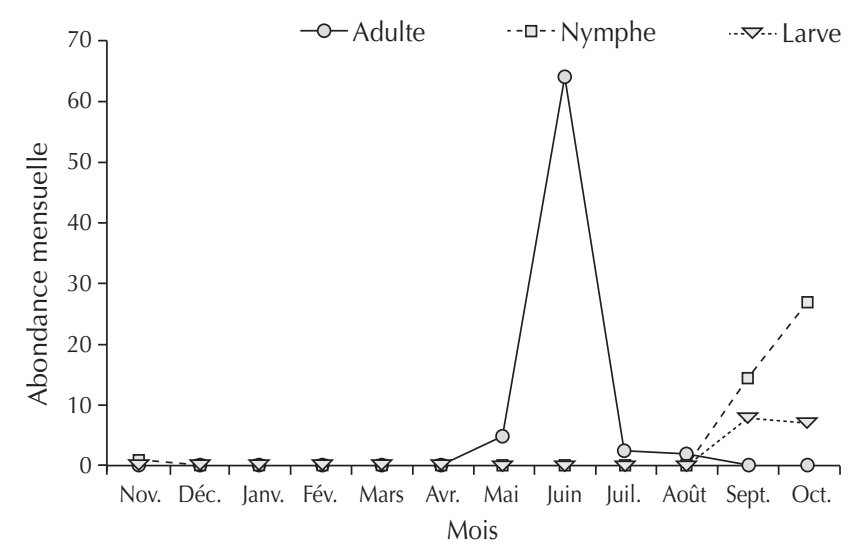

Figure 4 : fluctuations de l'abondance mensuelle de Hyalomma detritum detritum adulte, nymphe et larve dans la commune rurale de Sidi Bouker Elhaj dans la région du Gharb au Maroc. 
ongulés, surtout le mouton, la chèvre et le cheval, les bovins dans une moindre mesure. Les adultes de l'espèce seraient surtout actifs de fin janvier à début mai, les nymphes en septembre et octobre (17). En médecine vétérinaire, le rôle vecteur de $D$. marginatus est bien établi en Europe pour l'anaplasmose à Anaplasma ovis qui affecte les moutons et pour les piroplasmoses affectant les équidés (17).

Haemaphysalis sulcata a présenté un faible taux d'infestation. Son cycle est triphasique polytrope (8). Les tiques immatures se fixent principalement sur des reptiles comme les lézards, et les adultes sur les ongulés (ovins, caprins, bovins), mais aussi sur les chiens (8). Les adultes sont actifs en automne et en hiver entre octobre et mars. Les nymphes et les larves sont actives au printemps et en été (8).

Les traitements acaricides devraient être appliqués chez tous les bovins du troupeau, à certaines périodes de l'année, en tenant compte de l'activité saisonnière des tiques, de la charge parasitaire, et du taux d'infestation des animaux déterminé lors de l'étude. Les traitements peuvent être appliqués en hiver en ciblant les animaux qui présentent une infestation par I. ricinus, au printemps en visant les animaux infestés par $R$. bursa, Hyalomma lusitanicum et $R$. turanicus, et en été en s'intéressant aux animaux parasités par Hyalomma d. detritum

Les acaricides pourraient être appliqués sous forme d'injections sanguines ou d'aspersion sur les sites préférentiels de fixation des tiques sur les bovins. Bien entendu, toute stratégie rationnelle visant la lutte contre les tiques affectant le bétail se caractériserait par une approche diversifiée pour empêcher la sélection rapide de la résistance aux acaricides en alternant d'autres types de luttes : biologique, vaccins antitiques $(4,17)$. Des recherches devraient être menées également pour trouver des solutions alternatives à l'utilisation massive d'acaricides chimiques pour lutter contre les tiques (9).

\section{CONCLUSION}

La commune rurale de Sidi Boubker El Haj dans la région du Gharb est caractérisée par une couverture forestière abondante, constituée en majeure partie de chênes-lièges, d'eucalyptus, de cistes et de fougères, dont la faune est très diversifiée et comprend rongeurs, oiseaux, lézards et sangliers. Elle bénéficie d'une pluviométrie souvent supérieure à $600 \mathrm{~mm} / \mathrm{an}$, créant un milieu favorable à l'évolution de plusieurs espèces de tiques parasites du cheptel.

L'enquête menée dans cette région a permis de récolter sur des bovins neuf espèces de tiques appartenant à cinq genres. Les adultes d'I. ricinus ont présenté un mode d'activité unimodal hivernal, alors que ceux de $R$. bursa, Hyalomma lusitanicum et $R$. turanicus ont été actifs au printemps, et ceux de Hyalomma d. detritum en été. $R$. sanguineus, Hyalomma m. marginatum, D. marginatus et Haemaphysalis sulcata ont été moins abondants, leur présence sur le bétail pouvant être qualifiée de parasitisme occasionnel.

La dynamique des populations des tiques est essentielle pour la mise en place d'une stratégie efficace de lutte contre les tiques et les maladies qu'elles transmettent. Les traitements peuvent donc être utilisés chez les animaux les plus infestés par I. ricinus en hiver, par $R$. bursa, Hyalomma lusitanicum et $R$. turanicus au printemps, et par Hyalomma d. detritum en été.

\section{Remerciements}

Nous exprimons nos sincères remerciements aux professeurs Khadija El Kharrim et Driss Belghyti qui nous ont guidés tout au long de ce travail, à tous nos collègues du Laboratoire d'environnement et d'énergies renouvelables, et à toutes les personnes qui ont participé de près ou de loin à la réalisation de ce travail.

\section{BIBLIOGRAPHIE}

1. ARTHUR D.R., 1948. Some aspects of the ecology of the tick, Ixodes ricinus L. in Wales. Bull. entomol. Res., 39: 321-337.

2. BOUATTOUR A., 2002. Clé dichotomique et identification des tiques (Acari : Ixodidae) parasites du bétail au Maghreb. Arch. Inst. Pasteur, Tunis, $79:$ 43-50.

3. BOURDEAU P., 1993. Les tiques d'importance vétérinaire et médicale, $2^{\mathrm{e}}$ partie : principales espèces de tiques dures (Ixodidae et Amblymmidae). Point vét., $25: 27-41$.

4. BOWMAN A.S., NUTTALL P.A., 2008. Ticks biology, disease and control. Cambridge, UK, Cambridge University Press, 506 p.

5. CENTRE NATIONAL DE RECHERCHE SCIENTIFIQUE ET TECHNIQUE, 2010. Rabat, Maroc, Laboratoire de microscopie électronique à balayage. http://uatrs.cnrst.ma

6. DELLAC B., 1999. Maladies transmises aux bovins par les tiques. Action vét., 1478 : 19-24.

7. EDWARDS E.E., ARTHUR D.R., 1947. The seasonal activity of the tick, Ixodes ricinus L., in Wales. Parasitology, 38: 72-85.

8. ESTRADA-PENA A., BOUATTOUR A., CAMICAS J.L., WALKER A.R. 2004. Tiques d'importance médicale et vétérinaire : le Bassin méditerranéen. Icttd CD-ROM, 3-12, 12 p.

9. FAROUGOU S., KPODEKON M., TCHABODE D.M., YOUSSAO A.K.I, BOKO C., 2006. Abondance saisonnière des tiques (Acari : Ixodidae) parasites des bovins dans la zone soudanienne du Bénin : cas des départements de l'Atacora et de la Donga. Ann. Méd. vét., 150 : 145-152.

10. HAUT COMMISSARIAT AUX EAUX ET FORETS ET LA LUTTE CONTRE LA DESERTIFICATION, 2010. Etude d'aménagement de la forêt de Gharb. Rabat, Maroc, ministère de l'Agriculture et de la Pêche maritime, $70 \mathrm{p}$.

11. MARGOLIS L., ESCH G.W., HOLMES J.C., KURIS A.M., SHAD G.A., 1982. The use of ecological terms in parasitology. J. Parasitol., 68: 131133.

12. MOREL P.C., 1969. Contribution à la connaissance de la distribution des tiques (acariens, Ixodidae et Amblyommidae) en Afrique éthiopienne continentale. Thèse Doct., Université d'Orsay, Paris, France.

13. MOREL P.C., 2000 Maladies à tiques du bétail en Afrique. In : Chartier C., Itard J., Morel P.C., Troncy P.C., éds, Précis de parasitologie vétérinaire et tropicale. Cachan, France, Lavoisier Tech \& Doc, p. 456550 .

14. OFFICE REGIONAL DE MISE EN VALEUR AGRICOLE DU GHARB, 2009. Elevages en chiffres (2000-2009). Rabat, Maroc, ministère de l'Agriculture et de la Pêche maritime, 49 p.

15. OUHELLI H., 1985. Theilériose bovine à Theileria annulata : recherche sur la biologie des vecteurs Hyalomma spp. et sur les interactions hôte-parasite. Thèse Doct., Université de Toulouse, France.

16. OUHELLI H., 1988. Ecologie des Hyalomma (Ixodidae) parasites bovins au Maroc. Acta. Parasitol. Pol., 33 : 273-284.

17. PEREZ-EID C., 2007. Les tiques : identification, biologie, importance médicale et vétérinaire. Cachan, France, Lavoisier, 314 p. (Coll. Monographies de microbiologie)

18. SAHIBI H., RHALEM A., 2007. Tiques et maladies transmises par les tiques chez les bovins au Maroc. Transfert de technologie en agriculture. Bull. Inf. Liaison PNTTA, 151 : 1-4.

19. WALKER A.R., BOUATOUR A., CAMICAS J.L., ESTRADA-PENA A., HORAK I.G., LATIF A.A., PEGRAM R.G., PRESTON P.M., 2003. Ticks of domestic animals in Africa: a guide to identification of species. Edinburgh, UK, University of Edinburgh, 221 p. (Biosci. Rep.)

20. WALL R., SHEARER D., 2001. Veterinary ectoparasites: biology, pathology and control, 2nd Edn. Oxford, UK, Blackwell Science, 262 p.

21. YOUSFI-MONOD R., AESCHLIMANN A., 1986. Recherches sur les tiques (Acarina, Ixodidae) parasites des bovidés dans l'Ouest algérien Inventaire systématique et dynamique saisonnière. An. Parasitol. Hum. comp., $61: 341-358$.

Accepté le 14.02.2013 


\section{Summary}

Laamri M., El Kharrim K., Mrifag R., Boukbal M., Belghyti D. Population dynamics of cattle ticks in Gharb Region in Morocco

This study was conducted in the rural town of Sidi Boubker El Haj in Gharb Region, Morocco, from November 2009 to October 2010. The objective was to identify the various tick species collected on cattle, to monitor their seasonal dynamics and determine their abundance and infestation variability according to the age and sex of the hosts. Thirty cattle head were monitored and sampled monthly for a year. A total of 6899 ticks were collected. Identification revealed nine species belonging to five genera with the following prevalence: Rhipicephalus bursa $28.61 \%$, Ixodes ricinus $26.39 \%$, Hyalomma lusitanicum 23.33\%, Rhipicephalus turanicus 14.44\%, Hyalomma detritum detritum 13.06\%, Rhipicephalus sanguineus $1.39 \%$, Hyalomma marginatum marginatum 1.11\%, Dermacentor marginatus $1.11 \%$ and Haemaphysalis sulcata $0.83 \%$. I. ricinus was active in winter, R. bursa, Hyalomma lusitanicum and $R$. turanicus in spring, and Hyalomma d. detritum in summer. R. sanguineus, Hyalomma m. marginatum, D. marginatus and Haemaphysalis sulcata were less prevalent and their presence on cattle may be referred to as occasional parasitism. This study on tick population dynamics may help implement a strategy for tick control and tick-transmitted disease control, taking into account the parasite seasonal activity, parasitic loads, and infestation rates.

Keywords: Cattle - Metastigmata - Seasonal development Mite control - Gharb - Morocco.

\section{Resumen}

Laamri M., El Kharrim K., Mrifag R., Boukbal M., Belghyti D. Dinámica de las poblaciones de garrapatas parásitos de los bovinos de la región de Gharb en Marruecos

El presente estudio se llevó a cabo en la comunidad rural de Sidi Boubker El Haj, región de Gharb (Marruecos), de noviembre 2009 a octubre 2010. El objetivo fue el de identificar las diferentes especies de garrapatas encontradas en los bovinos, de seguir su dinámica estacional y de determinar su abundancia y la variabilidad a nivel de infestación según la edad y el sexo de los huéspedes. Treinta bovinos fueron objeto de seguimiento y muestras mensuales durante un año. En total, 6899 garrapatas fueron recolectadas. La identificación reveló la presencia de nueve especies pertenecientes a cinco géneros, con las siguientes prevalencias: Rhipicephalus bursa (28,61\%), Ixodes ricinus (26,39\%), Hyalomma lusitanicum (23,33\%), Rhipicephalus turanicus (14,44\%), Hyalomma detritum detritum (13,06\%), Rhipicephalus sanguineus (1,39\%), Hyalomma marginatum marginatum $(1,11 \%)$, Dermacentor marginatus $(1,11 \%)$ et Haemaphysalis sulcata $(0,83 \%)$. I ricinus presentó un modo de actividad unimodal invernal, mientras que $R$. bursa, Hyalomma lusitanicum y Rhipicephalus turanicus se activaron en la primavera y Hyalomma $d$. detritum en verano. R. sanguineus, Hyalomma m. marginatum, D. marginatus et Haemaphysalis sulcata fueron menos prevalentes, su presencia en el ganado puede ser calificada de parasitismo ocasional. Este estudio sobre la dinámica de las poblaciones de garrapatas puede ayudar a la implementación de una estrategia adaptada de lucha contra las garrapatas y las enfermedades que estas transmiten, teniendo en cuenta la actividad estacional de las garrapatas, de la carga parasitaria y de la tasa de infestación.

Palabras clave: Ganado bovino - Metastigmata - Desarrollo estacional - Control de ácaros - Gharb - Marruecos. 